Original Research Article

\title{
A study of risk factors for patients with Diabetic foot ulcer
}

\author{
Ravichandran K.S. ${ }^{1}$
}

${ }^{1}$ Dr. K.S. Ravichandran, Associate Professor, Department of General Surgery, Melmaruvathur Adhiparasakthi Institute of Medical Sciences, Tamilnadu, India.

Corresponding Author: Dr. K.S.Ravichandran, Associate Professor, Department of General Surgery, Melmaruvathur Adhiparasakthi Institute of Medical Sciences, Tamilnadu, India. E-mail: drksravichandranms@yahoo.com

\begin{abstract}
Background: Peripheral neuropathy and peripheral vascular diseases are the important causes of non-traumatic lower limb amputation. The risk of amputation among diabetic patients increases by two to four folds with the advancement of age and duration of diabetes.It has also been proven by many longitudinal epidemiological studies that among diabetic patients, the life time foot ulcer risk is about $25 \%$, thereby accounting for two thirds of all non-traumatic amputations. Clinical guidelines recommend that all patients with diabetes should be screened annually to establish their risk of foot ulceration. Aim: To ascertain the risk factors leading to amputation for patients with diabetic foot ulcer. Materials and method: The present study was conducted in the Department of General Surgery of Melmaruvathur Adhiparasakthi institute of medical sciences. Melmaruvathur. For our study, we selected 200 patients admitted to the surgical ward with diagnosis of diabetic foot ulcer. History of diabetic status of patient, whether patient was a undetected case or a known diabetic, if known the duration of the disease, whether patient was on regular or irregular treatment (diet/oral/drugs/insulin) were recorded. Lab tests of each patient were conducted for hemoglobin, TLC, DLC, ESR, blood urea, serum creatinine and blood sugar. All the cases were managed following conservative and surgical approach. Results: A total of 200 patients were present in the study group. The mean of the subjects was $49.28+6.88$ years. Out of 200 patients, 46 were females and 154 were males. We observed that 54 patients were undetected at the time of admission at hospital. Majority of patients $(n=46)$ had duration of diabetes from 5-10 years. 38 patients had duration of diabetes less than 4 years, 10 patients had duration of diabetes from 11-15 years. Most of the patients present with more than one lesion. Only major lesion is considered here. Ulcer was the major lesion seen in present series being present in 144 patients. Conclusion: We conclude that Routine foot care should be available to every patient with diabetes ideally, but the reality of most of these patient slack adequate knowledge and resources resulting in the absence of such care.
\end{abstract}

Keywords: Diabetes, Diabetic ulcer, Amputation, Foot ulcer

\section{Introduction}

Few of the well-known complications of diabetes are Peripheral neuropathy (PN) and peripheral vascular disease (PVD)[1] Patients with PN and PVVD lack the conventional symptoms, but are still considered to be at high risk for occurrence of foot complications [2-4]. PN and PVD are the main causes of non-traumatic lower limb amputation [5]. The risk of ulceration and amputation among diabetic patients increases by two to four folds with the progression of age and duration of diabetes regardless of the type of diabetes [6]. It has also been proven by many longitudinal epidemiological studies that among diabetic patients, the life time foot ulcer risk is about $25 \%[7,8]$. Thereby accounting for

Manuscript Received: $4^{\text {th }}$ February 2019

Reviewed: $14^{\text {th }}$ February 2019

Author Corrected: $20^{\text {th }}$ February 2019

Accepted for Publication: $26^{\text {th }}$ February 2019 two thirds of all non-traumatic amputations. Clinical guidelines recommend that all patients with diabetes should be screened annually to establish their risk of foot ulceration [8]. Diagnostic tests and physical signs that detect peripheral neuropathy (biothesiometry, monofilaments and absent ankle reflexes), and those that detect excessive plantar pressure (peak plantar pressure and joint deformity) were all significantly associated with future diabetic foot ulceration [9,10]. Hence the present study is planned to assess the risk factors for patients suffering diabetic foot ulcer.

\section{Materials and Method}

Settings-The present study was conducted in the Department of General Surgery of the Melmaruvathuradhiparasakthi institute of medical sciences and 


\section{Original Research Article}

research. It is a medical college, a tertiary care center, catering to a population of around five lakh people, mostly rural population.

Type of study- It is a prospective study, analyzing the different factors associated with diabetesleading to ulcers of the legs.

Ethical clearance-The ethical clearance for the protocol of study was obtained from the ethical committee of the institute.

Inclusion criteria- For the study we selected 200 patients admitted to the surgical ward with diagnosis of diabetic foot. The diagnosis was confirmed by detailed history and clinical examination. An informed written consent was obtained from each patient after explaining to them the procedure of the study.

Exclusion criteria- Patients with end stage renal disease, patients on regular dialysis. Patients with coronary heart disease, Patients with history of tuberculosis or, leprosy, patients with unstable parameters, were excluded from the study.

Statistical methods- History of diabetic status of patient was recorded. Whether the patient was an undetected case or a known diabetic, and, if known, the duration of the disease, was noted whether patient was on regular or irregular treatment (diet/oral/drugs/ insulin) was also recorded. Lab tests of each patient were conducted for hemoglobin, TLC, DLC, ESR, blood urea, serum creatinine and blood sugar. Values were evaluated periodically Whenever vascular insufficiency was detected in lower limbs, serum cholesterol ECG and Doppler was done. In all the diabetic foot cases, pus was sent for culture and sensitivity examination before starting antibiotics. All the details were carefully recorded and analysed.

Surgical methods- All the cases were managed following conservative and surgical approaches, where ever necessary. Appropriate Antibiotics and analgesics were prescribed for infection and inflammation. Proper dressings were done.

\section{Results}

The statistical analysis of the data was done using SPSS software for windows. The significance of the data was checked using Chi-square test and Student's t-test. A p-value $<0.05$ was predetermined to be statistical significant. The mean of the subjects was $49.28+6.88$ years. Out of 200, 46 were females and 154 were males. Table 1 shows the frequency of patients with different duration of diabetes. We observed that 54 patients were undetected at the time of admission at hospital. Majority of patients $(n=92)$ had duration of diabetes from 5-10 years. 38 patients had duration of diabetes less than 4 years, 10 patients had duration of diabetes from 11-15 years. Only 2 patients in the group had diabetes from 21-25 years.

Table-1: Frequency of patients with different duration of diabetes.

\begin{tabular}{|c|c|}
\hline Duration of diabetes & Number of patients \\
\hline Undetected & 54 \\
\hline$<4$ years & 38 \\
\hline $5-10$ years & 92 \\
\hline $11-15$ years & 10 \\
\hline $16-20$ years & 4 \\
\hline $21-25$ years & 2 \\
\hline Total & $\mathbf{2 0 0}$ \\
\hline
\end{tabular}

Table-2: Incidence of different type of lesions in study group.

\begin{tabular}{|c|c|}
\hline Type of lesion on foot & No. of cases \\
\hline Gangrene & 58 \\
\hline Cellulitis & 94 \\
\hline Abscess & 32 \\
\hline Ulcer & 144 \\
\hline
\end{tabular}

Table- 2 shows the incidence of different type of lesions in study group. The different types of lesions included cellulitis, abscess, ulcer and gangrene. Most of the patients present with more than one lesion. Only major lesion is considered here. Ulcer was the major lesion seen in present series being present in 144 patients. While abscess seen in 32 patients, was the least common lesion. 


\section{Original Research Article}

\section{Discussion}

Diabetes mellitus- Diabetes mellitus is a worldwide disease.It affects all ages, It affects both male and female equally. Almost all races are affected by diabetes. Diabetes mellitus has reached epidemic properties worldwide as we enter the new millennium. The world health organization has commented there is "an apparent epidemic over the next decade the projected number will exceed 200 million, Diabetes affects the entire body, from head to toe. Diabetic neuropathy, Diabetic nephropathy, Diabetic retinopathy, and coronary ischaemia are some of the common problems associated with diabetes. However, the most common one is foot ulcer.

Diabetic foot is a serious complication of diabetes mellitus when compared with people without diabetes. Foot ulcers are significant complications of diabetes mellitus and often precede lower extremity amputation. Recurrence of the foot infection was common among India diabetic patients about 52\%. 6 Infection and gangrene of the lower extremities are the most common lesions requiring hospitalization in diabetes and are a major cause of morbidity [4].

In the present study, we observed that diabetic foot ulcer was most common in the patients having diabetes since 8-10 years. Patients with longstanding diabetes will definitely develop foot ulcers at some point of time. Also, the diabetic foot ulcer was most common lesion observed in patients. Few patients had callus, corn foot, abscess, eczema etc. The results were compared with previous studies and were found to be consistent. Shahbazian $\mathrm{H}$ et al assessed diabetic foot ulcer risk factors according to International Working Group on the Diabetic Foot (IWGDF) consensus.

Based on the IWGDF criteria, they divided all the referral patients into four study groups; patients without neuropathy, patients with neuropathy, patients with vascular disease associated neuropathy and patients with foot ulcer. 53.8 years was the mean age of the patients in their study group. They analyzed a total of 400 patients out of which 269 were females, while the remaining 161 were males.

Disturbance in the sense of vibration was observed in 23 percent of the patients in their study, while fall in sensitivity to monofilaments was seen in 26 percent of the patients. In 17 percent of the patients, they observed a decrease in pain sensation. In 6 percent of the patients, they observed abnormality in ankle brachial index
(ABI). Past history of prior ulcer was seen in 7 percent of the patients of their study. From the results, they concluded that advancing age along with diabetes duration and diabetic retinopathy increases the risk for the development of diabetic ulcer [11].

In another study conducted by $\mathrm{Wu} \mathrm{L}$ et al, the authors determined the prevalence of various risk factors responsible for occurrence of diabetic foot in patients with diabetes. They retrospectively evaluated a total of 296 patients who were admitted to the tertiary hospital because of diabetes. A questionnaire was framed and was made to be filled by all the patients. They also assessed their foot along with presence of absence of peripheral sensory neuropathy (PSN) and peripheral arterial disease (PVD). They observed foot deformity in 124 patients with the most prevalent abnormality being hallux valgus, which was observed in 65 percent of the patients of their study. From the results, they concluded that risk factors for foot ulceration and lack of fool care knowledge was rather common in a hospital-based diabetic population, emphasizing the importance of implementing simple and affordable screening tools and methods to identify high-risk patients and providing foot care education for them $[11,12]$.

In another study conducted by Sarinnapakorn V et al, the authors evaluated the overall prevalence of diabetic foot ulcers. From the results, they concluded that diabetic patients are at intermediate for the development of diabetic foot ulcer. They also stressed that for the classification of the diabetic patients on the basis of risk factors, it is necessary to classify patients. Nyamu PN et al, in another study, evaluated the prevalence rate of patients with diabetic foot ulcers and the risk factors.

They evaluated a total of 1788 diabetic patients and observed that in approximately four to five percent of the patients had diabetic foot ulcer. They observed presence of diabetic foot ulcer in patients with comparatively longer duration of diabetes. From the results, they concluded that the risk factors of diabetic foot ulcers were poor glycemic control, diastolic hypertension, dyslipidaemia, infection and poor selfcare $[13,14]$.

In our study of 200 patients, we find majority of the patients with ulcers belong to the chronic diabetes group. The pathological changes in the nervous system lead to sensory impairment and peripheral neuropathy. Similarly, the changes in the blood vessel walls lead to 


\section{Original Research Article}

narrowing of the lumen and gradual ischaemia develops. These changes are more visible in the lower limbs. Diastolic hypertension is seen in many of the patients with diabetic foot ulcers. Peripheral neuropathy was seen in more than 80 percent of the study group.

Folic acid deficiency and Vitamin B12 deficiency have been documented in many studies. In our study, 68 percent were having sub optimal levels of B12. Most of our population are not using shoes. In our study, 56 percent were using normal open type footwear. The recurrence rate of ulcer was high among them, when compared to those using shoes.

Another important finding was that diabetic persons with chronic smoking, tend to come with recurrent ulcers. inspite of adequate glycemic control. It may be well explained by the vascular ischaemia of the extremities. We have also noted, Iron deficiency anaemia was seen in 86 patientsand Hypoproteinemia seen in 36 patients. Ulcer healing was very much delayed in these patients with anaemia and hypoproteinemia. Patients on regular insulin medication were less affected with leg ulcers, when compared with patients on oral hypoglycemic agents. Patients on OHA develop diabetic foot ulcer earlier than those on Insulin,

\section{Conclusion}

Diabetic foot ulcer is a rapidly increasing entity. In our study, the important risk factors were found to be poor glycemic control,peripheral neuropathy, and poor podiatric care. The same has been evaluated in many publications in the past.

Within the limitations of present study, we conclude that Routine foot care should be available to every patient with diabetes. Adequate glycemic control to be achieved with insulin, All health care facilities should have a podiatric wing and trained healthcare workers.

Conflict of interest: None declared.

Funding: Nil, Permission from IRB: Yes

\section{References}

1. Walters DP, Gatling W, Mullee MA, et al. The prevalence, detection, and epidemiological correlates of peripheral vascular disease: a comparison of diabetic and non-diabetic subjects in an English community. Diabet Med. 1992 Oct;9(8):710-5.

2. Pecoraro RE, Reiber GE, Burgess EM. Pathways to diabetic limb amputation. Basis for prevention. Diabetes Care. 1990 May;13(5):513-21.
3. Adler AI, Boyko EJ, Ahroni JH, Smith DG. Lowerextremity amputation in diabetes. The independent effects of peripheral vascular disease, sensory neuropathy, and foot ulcers. Diabetes Care. 1999;22: 1029-35. doi: 10.2337/diacare.22.7.1029.

4. Lehto S, Rönnemaa T, Pyörälä K, Laakso M. Risk factors predicting lower extremity amputations in patients with NIDDM. Diabetes Care. 1996;19:607-12. doi: 10.2337/diacare.19.6.607.

5. Trautner C, Haastert B, Giani G, et al. Incidence of lower limb amputations and diabetes. Diabetes Care. 1996 Sep;19(9):1006-9.

6. Siitonen O, Niskanen L, Laakso M, Siitonen J, Pyorala K. Lower-extremity amputations in diabetic and nondiabetic patients. A population-based study in eastern Finland. Diabetes Care. 1993;16:16-20. doi: 10.2337/ diacare.16.1.16.

7. Unwin N. Epidemiology of lower extremity amputation in centres in Europe, North America and East Asia DOI: 10. 1046/j. 1365-2168. 2000.0 1344.x

8. International Working Group on the Diabetic Foot. Epidemiology of Diabetic Foot Infections in a Population Based Cohort. Noordwijkerhout, The Netherlands; 2003.

9. Lavery LA, Armstrong DG, Wunderlich RP, et al. Diabetic foot syndrome: evaluating the prevalence and incidence of foot pathology in Mexican Americans and non-Hispanic whites from a diabetes disease management cohort. Diabetes Care. 2003 May; 26 (5): 1435-8.

10. Boulton AJ, Vileikyte L, Ragnarson-Tennvall G, et al. The global burden of diabetic foot disease. Lancet. 2005 Nov 12;366(9498):1719-24.DOI:10.1016/ S0140-6736(05)67698-2

11. Shahbazian H, Yazdanpanah L, Latifi SM. Risk assessment of patients with diabetes for foot ulcers according to risk classification consensus of International Working Group on Diabetic Foot (IWGDF). Pak J Med Sci. 2013 May;29(3):730-4.

12. Wu L, Hou Q, Zhou Q, et al. Prevalence of risk factors for diabetic foot complications in a Chinese tertiary hospital. Int J Clin Exp Med. 2015 Mar 15;8(3): 3785-92. eCollection 2015. 


\section{Original Research Article}

13. Sarinnapakorn V, Sunthorntepwarakul T, Deerochanawong C, et al. Prevalence of Diabetic Foot Ulcers and Risk Classifications in Type 2 Diabetes Mellitus Patients at Rajavithi Hospital. J Med Assoc Thai. 2016 Feb; 99 Suppl 2:S99-105.
14. Nyamu PN, Otieno CF, Amayo EO, et al. Risk factors and prevalence of diabetic foot ulcers at Kenyatta National Hospital, Nairobi. East Afr Med J. 2003 Jan; 80(1):36-43.

\section{How to cite this article?}

Ravichandran K.S. A study of risk factors for patients with Diabetic foot ulcer. Surgical Update: Int J surg Orthopedics. 2019; 5(1):41-45.doi:10.17511/ ijoso.2019.i01.07.

Diabetes, Diabetic ulcer, Amputation, Foot ulcer 